\title{
Les dynamiques industrielles en Pays de Chaumont (Haute-Marne) - De la tradition artisanale coutelière aux implants chirurgicaux et au Cluster Nogentech
}

The industrial dynamics in the Pays de Chaumont (Haute-Marne) - From artisanal cutlery tradition to surgical implants and to cluster Nogentech

Daniel Ricard

\section{(2) OpenEdition}

\section{Journals}

Electronic version

URL: http://journals.openedition.org/rge/5598

ISSN: 2108-6478

\section{Publisher}

Association des géographes de l'Est

Printed version

Date of publication: 1 October 2015

ISSN: 0035-3213

\section{Electronic reference}

Daniel Ricard, « Les dynamiques industrielles en Pays de Chaumont (Haute-Marne) - De la tradition artisanale coutelière aux implants chirurgicaux et au Cluster Nogentech », Revue Géographique de l'Est [Online], vol.55 / n³-4 | 2015, Online since 14 October 2015, connection on 08 September 2020. URL : http://journals.openedition.org/rge/5598

This text was automatically generated on 8 September 2020

Tous droits réservés 


\section{Les dynamiques industrielles en Pays de Chaumont (Haute-Marne) - De la tradition artisanale coutelière aux implants chirurgicaux et au Cluster Nogentech}

The industrial dynamics in the Pays de Chaumont (Haute-Marne) - From artisanal cutlery tradition to surgical implants and to cluster Nogentech

\section{Daniel Ricard}

Parler d'industrie, qui plus est dans la France de l'Est, incline plutôt à s'inscrire dans l'analyse d'un processus de déclin. Déclin de l'industrie nationale, encore aggravé par la crise de 2008 ; déclin plus spécifique des vieilles régions industrielles de ces territoires ; localisation enfin dans des milieux de faible densité éloignés des métropoles, dans la "diagonale du vide». Bref, tout semble réuni pour se livrer à une géographie de la crise, un positionnement confirmé par le spectaculaire recul des emplois de la coutellerie nogentaise. Très vite cependant, la réalité apparaît bien différente au géographe qui arpente ces régions et constate une reconversion plutôt réussie vers le matériel médical et les prothèses chirurgicales, pour une industrie qui conserve un rôle clef dans l'économie et la vie locale, surtout vers Nogent.

2 C'est là le cœur de notre réflexion, alimentée par le travail bibliographique et de nombreuses enquêtes conduites en 2014/2015 auprès des industriels et de leurs structures d'encadrement. L'analyse exige aussi de prendre un certain recul, à la fois pour faire appel aux enseignements de l'histoire et la replacer dans le cadre plus vaste de l'industrie nationale et internationale.

Revenons au territoire investigué. Ce texte s'intéresse au Pays de Chaumont, fort de 154 communes et taillé pour l'essentiel dans les plateaux calcaires froids et caillouteux du Barrois. Il occupe la partie centrale du département de la Haute-Marne et est désormais 
structuré par cinq communautés de communes et la communauté d'agglomération de Chaumont. Le Pays est un territoire fortement rural ${ }^{1}$, peu peuplé (66 007 habitants en 2011) et surtout en recul démographique structurel : - $6 \%$ entre 1975 et 1999, - 4,9\% encore de 1999 à 2011 (INSEE). Les activités tertiaires dominent, surtout à Chaumont, le prototype de la ville administrative. L'agriculture se partage entre l'élevage laitier, encore bien présent et la céréaliculture, qui s'appuie sur de vastes exploitations hélas confrontées au faible potentiel des terres. Elle souffre de la médiocrité de l'aval, qui se résume à la grosse fromagerie Bongrain d'Illoud (Caprice des dieux) et aux énormes silos de céréales qui parsèment la campagne. C'est en fait l'industrie, la métallurgie, qui fait l'originalité de ce territoire, en liaison notamment avec un fort ancrage historique. Elle sera au cœur de notre recherche.

Fig. 1 : Le pays de Chaumont, carte de localisation

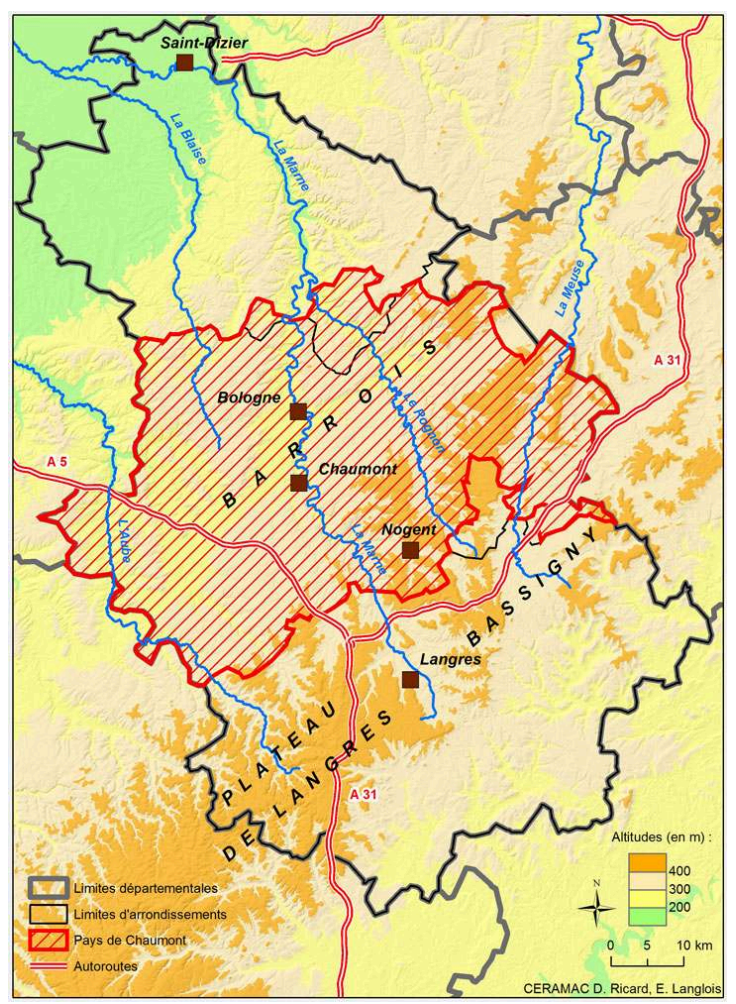

\section{Une industrie en rupture avec la dynamique récessive nationale}

4 L'industrie française s'inscrit de nos jours dans une dynamique clairement récessive, commune à presque tout le continent, monde germanique exclu. Les expressions de ce recul sont connues : baisse de l'emploi depuis les années 1970, baisse de la production dans nombre de branches industrielles... Les facteurs explicatifs sont connus (rapport Gallois, 2012), toujours multiples et souvent cumulatifs : coût de la main-d'œuvre, au moins par rapport au positionnement produit, offre parfois mal adaptée à la demande, déficit en matière de recherche et de formation des travailleurs, faiblesse du tissu des PME, engagement commercial insuffisant, notamment à l'exportation... La crise de 2008 
a révélé les faiblesses structurelles de cette industrie, surtout dans certains métiers et surtout dans certaines régions, notamment du Nord et du Nord-Est de la France.

Fig. 2 : Evolution comparée du taux de chômage de 2003 à 2014 (en \%)

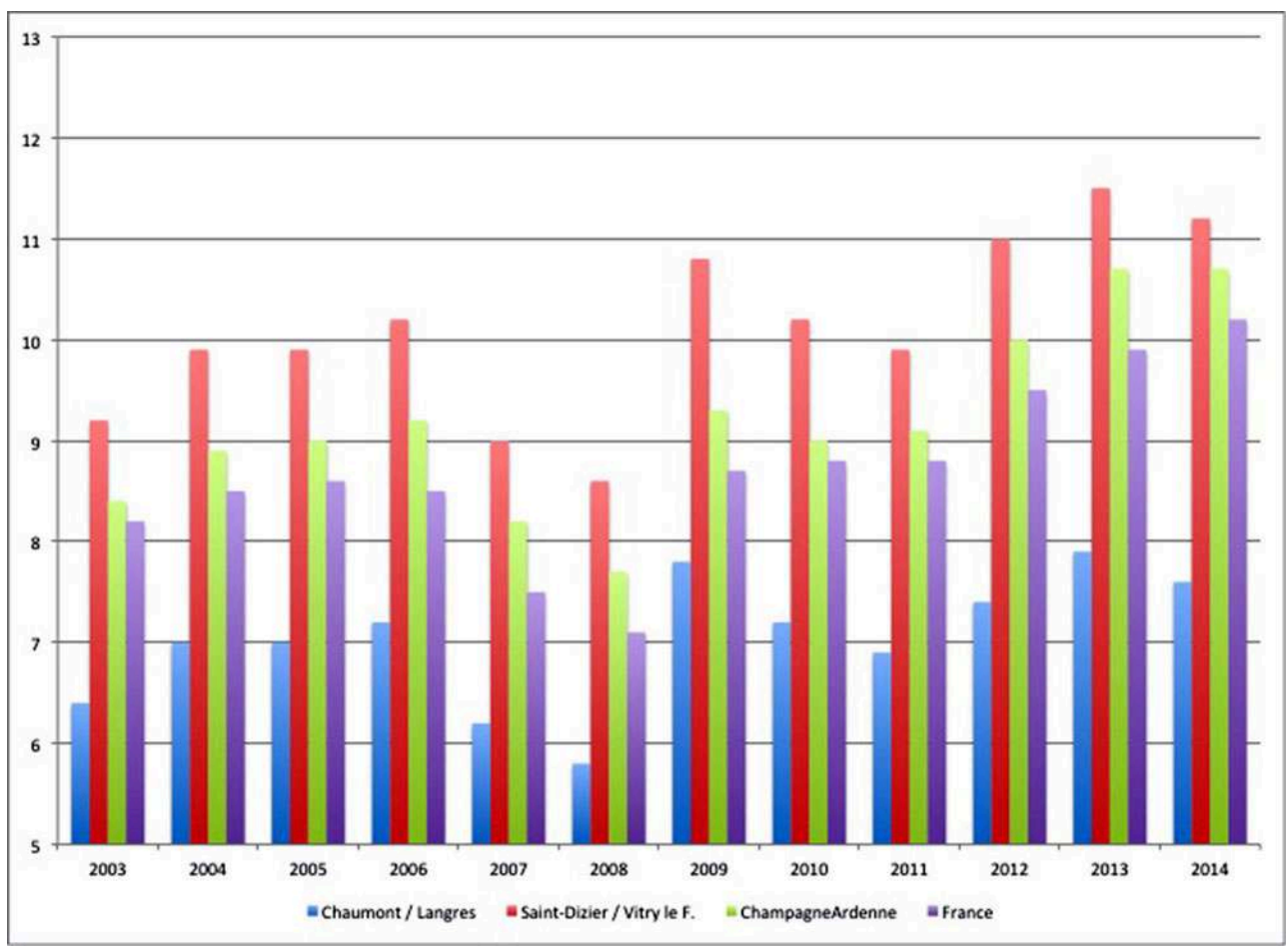

Source : d'après données INSEE

5 Comment positionner le Chaumontais dans cet environnement ? Au-delà du problème de la délimitation du territoire d'étude ${ }^{2}$, le constat principal est bien celui d'une meilleure résistance de l'industrie locale. Les pertes d'emploi y restent contenues sur le temps long, la disparition massive de l'activité coutelière ${ }^{3}$ ayant été grossièrement compensée par les nouveaux emplois liés au secteur médical. Quant au chômage, il est plus faible ici qu'ailleurs ${ }^{4}$, que ce soit par rapport au Nord du département, à la Région Champagne-Ardenne ou à la France métropolitaine (Fig. 1). Certes, la crise l'a fait bondir de 5,8 à 7,8 \% entre 2008 et 2009, mais le bassin d'emploi de Chaumont / Langres a mieux résisté depuis, puisque le taux de chômage recule de 0,3 \% entre 2009 et 2014 alors qu'il progresse de 1,3\% en France et de 1,7 \% dans la Région. Résultat, il est aujourd'hui inférieur de 2,6 points à la moyenne nationale, un écart considérable. L'INSEE classe d'ailleurs Chaumont / Langres dans le quart supérieur des zones d'emploi les plus performantes sur ce critère, au 60e rang sur 321. L'industrie joue à l'évidence son rôle dans cette dynamique globale.

Quelles sont les causes de cette dynamique industrielle? Et où est la différence avec les autres territoires? Les enquêtes de terrain, d'un grand intérêt pour l'analyse, soulignent alors l'importance du savoir-faire des forgerons haut-marnais, la capacité d'innovation de ce territoire (ce qui renvoie au rôle des hommes et à l'action de quelques leaders) et l'aptitude des entreprises à s'inscrire positivement et efficacement dans la mondialisation. 


\section{L'arrière-plan historique : la tradition forgeronne}

7 Comprendre cette dynamique industrielle conduit à interroger le passé, celui d'une Haute-Marne qui fut longtemps le premier département sidérurgique de France! La métallurgie, qui remonte au XIIe siècle, s'appuie alors sur des ressources locales bien présentes (Collectif, 1997). Les gisements ferrifères sont d'assez faible teneur, mais facilement accessibles. Le charbon de bois vient des immenses forêts de feuillus de la région. Reste l'énergie, que l'on puise dans les nombreux cours d'eau : Marne, Rognon, Blaise...

8 En un temps où le poids du déterminisme est essentiel, on constate l'émergence d'une intense activité métallurgique. Sur le plan géographique, l'avantage va au Nord du département, aux vallées de la Blaise (Wassy, Dommartin-le-Franc...), du bas Rognon (Doulaincourt, Saucourt), du Rongeant (Poissons) et surtout de la Marne en aval de Froncles. La présence de limonites crétacées riches en fer explique en bonne partie cette géographie particulière dont les centres principaux sont à Saint-Dizier et à Wassy (Collectif, 1997).

9 Cette métallurgie est bousculée au XIXe siècle par le progrès technique et l'arrivée des fourneaux dits anglais. La Haute-Marne, dépourvue de houille, tente de résister en adoptant (années 1850) un procédé intermédiaire fonctionnant avec un mélange de charbon de bois et de charbon de terre (Collectif, 1997). Elle met aussi en avant la qualité de ses minerais et donc de ses produits finis, recherche la valeur ajoutée (fonte moulée...) et se concentre spatialement autour de Saint-Dizier. Symbole de cette résistance, la Haute-Marne est toujours le premier département métallurgique français en 1859 (Collectif, 1997) et la production de fonte y atteint son maximum tardivement, en 1866, avec 102400 tonnes.

10 Mais l'activité est condamnée par le progrès technique et l'industrialisation, par le Traité de commerce Franco-anglais de 1860 aussi. Le département se fait doubler par la Moselle cette année-là, le Nord en 1861, puis la Saône-et-Loire et surtout la Lorraine qui, grâce à ses énormes ressources ferrifères et au nouveau procédé Thomas et Gilchrist (années 1880) peut passer au stade industriel. Nos forgerons investissent alors en Lorraine pour contrôler l'approvisionnement, s'appuient sur la réputation de leurs produits et se montrent agressifs sur le plan commercial. La métallurgie souffre donc mais s'adapte, ce qui explique la permanence jusqu'à nos jours d'importantes sociétés orientées vers la fonderie ou la forge.

11 Le Pays de Chaumont relève aussi d'une autre dynamique venue de Langres où s'était développée une importante corporation des couteliers. Des difficultés d'accès à l'eau (on est sur une butte), des installations intra-muros bruyantes et des corporations très rigides conduisirent toutefois à interdire l'installation de nouveaux couteliers à moins de quatre lieues du bourg au XVIIe siècle (Savouret, 1983). Dès lors, les fabricants s'expatrient, à l'image des Belligné, une grande famille de la coutellerie, et s'installent dans un vaste territoire qui va de Langres à Chaumont et à Neufchâteau, à cheval sur le Barrois et le Bassigny. Nogent-le-Bas, le long de la Traire, constitue le centre géographique de la migration (Savouret, 1983).

Cette région coutelière connaît son apogée dès $1850 / 1880$, alors que ne restent plus à Langres que des négociants (Savouret, 1983). On y constate l'individualisation précoce de spécialisations locales : sécateurs à Dampierre, ciseaux et scalpels à Nogent... Une 
partie de la production est réalisée à domicile par des paysans qui s'orientent souvent vers un travail donné (estampage...) et vendent aux négociants le dimanche au marché. Des artisans prennent quant à eux en charge la réalisation des pièces entières. Enfin, dans la seconde moitié du XIXe siècle, des entreprises embauchent des salariés et développent le travail à façon. La coutellerie, et plus encore la cisellerie, deviennent un secteur économique clef (Savouret, 1983).

13 L'industrie de Haute-Marne s'appuie donc sur deux piliers complémentaires. Au Nord, vers Saint-Dizier et dans les vallées de la Marne et de Blaise, on s'oriente vers la fonderie. Au Sud, la forge et la coutellerie dominent, avec des entreprises plus petites mais plus nombreuses, mieux réparties dans le territoire, à la faveur d'un fort essaimage en milieu rural.

\section{La coutellerie nogentaise, de l'apogée au repli structurel}

dans le «Tour de la France par deux écoliers » des enfants de la Troisième République, publié en 1877. On est là dans une logique de spécialisation territoriale fondée sur des savoir-faire (bien plus que sur la ressource locale), avec émergence progressive d'une réputation liée aux produits locaux, le tout dans le cadre d'un marché national protégé. Ce systène entre toutefois en récession dès la fin du $x$ que M. Savouret recensait sous le Second Empire ne sont plus que 1750 en 1975, puis 80 environ en 2015, chez une petite dizaine d'artisans (20 à 25 emplois), Daguin estampage à Is-en-Bassigny (14 salariés) et Nogent*** / Kimex (43 personnes). Outre la coutellerie, on fabrique aussi des ciseaux (la spécialisation initiale du bassin nogentais) et des pinces à épiler (Couturier à Sarrey). Certains se sont reconvertis pour tout ou partie, comme Eloi Pernet à Nogent ${ }^{5}$. La société Nogent*** de Biesles et sa filiale Kimex, spécialisée dans les couteaux non fermants, a atteint quant à elle une dimension semiindustrielle. Quelques artisans ont investi, à l'inverse, dans le haut de gamme, comme Liiri à Foulain (un ciselier Meilleur Ouvrier de France) et Mongin à Biesles (couteaux sur mesure, distribution chez Hermès...). Mais la profession a été récemment victime de la reprise ratée de Dussaussay de Nogent ${ }^{6}$. La fabrication de couverts fait vivre quant à elle une affaire familiale qui regroupe les Couverts de Darney (Vosges, produits en acier inox), les Couverts de Mouroux (Nogent, produits argentés) et Mécadis (Mandres, argenture, traitement de surfaces). On sort toutefois là de la coutellerie proprement dite qui se caractérise, elle, par l'utilisation de l'acier trempé, le durcissement par la trempe donnant le tranchant spécifique aux produits.

En fait, le déclin structurel de la coutellerie / cisellerie s'explique par plusieurs facteurs cumulatifs.

- Le premier, extérieur à la région et à la profession, vient de la mutation de cette activité dans le domaine productif et commercial. La grande distribution a fait entrer la coutellerie dans la catégorie des productions standardisées soumises à une forte pression sur les prix et la recherche de produits pouvant passer au lave-vaisselle renforce ce processus de standardisation / banalisation. Le développement de la concurrence étrangère renvoie quant à elle à l'ouverture des frontières. Pour cette production gourmande en main-d'œuvre, l'avantage va rapidement à l'Asie, au Pakistan

Revue Géographique de l'Est, vol.55 / n³-4 | 2015 
surtout et on assiste alors à un processus d'envahissement du marché par des produits à bas prix importés.

Le second groupe d'explications renvoie à un milieu local incapable de s'adapter. On constate bien vite cet « individualisme forcené » qu'évoque un de nos interlocuteurs : les artisans ne se connaissent guère, échangent peu. Certes, il y a la Confrérie du Bassin Coutelier Nogentais, qui a d'ailleurs lancé la marque « Le Nogentais» (2011)... mais elle n'est exploitée que par un seul fabricant. Il n'y a pas en revanche de vraie structure interprofessionnelle susceptible de donner une nouvelle impulsion au métier et au produit. Ajoutons à cela le problème de la transmission des savoir-faire. Les couteliers peinent à former des apprentis, les formations spécifiques en collège sont confrontées au recul des candidats et les difficultés de recrutement sont réelles. Or, une hypothétique relance par le haut de gamme ne peut que passer par l'expression de ces savoir-faire...

19 La coutellerie cisellerie nogentaise est donc sur la mauvaise pente : les effectifs s'étiolent, les artisans vieillissent, les reprises sont difficiles et l'absence de dynamique collective est pénalisante. Or, il y a derrière ces métiers de vrais savoir-faire, surtout dans la fabrication de ciseaux, dont certains sont des œuvres d'art. Reste le processus de patrimonialisation, porté par le Musée de la coutellerie, créé en 1991 dans l'ancien local de la coutellerie Pelletier à Nogent, mais hélas ouvert en été seulement à cause de la faible fréquentation touristique du bourg.

\section{La recomposition de l'industrie métallurgique}

Cette activité coutelière et métallurgique va toutefois connaître une réorientation progressive, depuis la guerre, vers des créneaux novateurs et porteurs, sous l'impulsion de quelques entrepreneurs dont il faut retracer le parcours.

21 Les Landanger sont à la base de cette réorientation. "C'est la clef », nous affirme-t-on. Le couple, artisan ciselier et coutelier installé à Mandres-la-Côte en 1947, se lance dès l'année suivante dans la fabrication d'instruments chirurgicaux et innove en courtcircuitant les grossistes pour démarcher directement les chirurgiens. L'affaire se développe très vite sur ce créneau très porteur et s'installe à Chaumont où elle passe au stade industriel dès les années 1960, puis aborde la fabrication des prothèses de hanche (puis d'autres articulations), ce qui conduit à usiner de nouveaux matériaux (titane, cobalt, alumine...) et à implanter des succursales commerciales à l'étranger. Landanger assure le cycle complet de production en élaborant les tiges forgées ainsi que les rotules recouvertes de matériaux spécifiques, cette dernière opération se nourrissant d'un gros travail de recherche et conduisant au dépôt de nombreux brevets. L'affaire est vendue en 1997 et est désormais contrôlée par Greatbatch, le groupe américain fondé par l'inventeur du pacemaker.

La saga familiale ne s'arrête pas là. L'un des fils, qui avait monté sa propre affaire d'instruments chirurgicaux à Chaumont dès 1968, la cède en 1993 à l'Allemand Aesculap, lui-même repris en 1998 par B. Braun, une grosse PME familiale de la région de Kassel ( 5 milliards d'euros de chiffre d'affaires). Le site compte 120 salariés et livre 40000 prothèses de genou par an, exportées à $98 \%$ \% . L'entrepreneur reprend la même année Eloi Pernet (revendu en 2010 à la Caisse des Dépôts) et créé en 2003 C2F Implants, une société qui conçoit, développe et commercialise des implants orthopédiques dont il sous-traite la fabrication. Un autre fils avait conservé en 1997 le secteur des 
instruments chirurgicaux (50 personnes) qui n'intéressait pas le repreneur. Il développe l'affaire puis rachète en 2005 la société parisienne concurrente Delacroix Chevalier. L'actuelle usine de la Z.I. La Vendue à Chaumont compte aujourd'hui 150 salariés. Reste la nouvelle génération, avec un petit-fils qui intervient aussi sur le créneau des instruments chirurgicaux à chaumont.

L'autre acteur clef de la mutation est Bernard Marle, fils d'un producteur d'outils et de matrices pour la coutellerie d'Odival, aux portes de Nogent. Marle reprend les 11 salariés en 1978 et réoriente l'affaire deux ans plus tard vers le médical, "par passion pour les implants ", en maîtrisant progressivement la difficile technique du forgeage du titane. La croissance est spectaculaire, sur un créneau très porteur et se nourrit d'une baisse spectaculaire des coûts de production, qui seront divisés par dix en 35 ans! L'entreprise fabrique aujourd'hui 600000 prothèses (dont 500000 de hanche) et emploie 180 personnes, plus 60 dans sa filiale Haute-Marne Parachèvement de Sarrey, reprise en 2001. B. Marle se désengage toutefois en 2009 en cédant $80 \%$ de ses parts au fonds américain Carlyle. Le Groupe compte aujourd'hui 5 sites en France (fonderie ATS à Alès, usinage Sferic à Ménars (41), usinage revêtement à Sorbiers (42), polissage à Sarrey et prothèses à Nogent). Notons que Marle n'a jamais contrôlé l'ensemble de la filière, à la différence de Landanger, en demeurant producteur de pièces en métal vendues aux fabricants de prothèses. Il se considère donc comme un "sous-traitant des fabricants", une situation qui n'interdit manifestement pas une rentabilité spectaculaire, puisque le site nogentais offre un résultat net de 5,5 millions d'euros en 2012 pour un chiffre d'affaires de 32,9 millions (dont $47 \%$ à l'export)!

Le troisième acteur de la mutation est à rechercher à Nogent-le-Bas, avec les Deguy, propriétaire des Forges de Courcelles, fondées en 1878 par les familles Wichard et Conge, leurs ancêtres ${ }^{8}$. Les deux frères Deguy, qui accèdent aux commandes en 1950 / 1952, sont à la base du développement de cette forge qui, à l'origine, fabriquait surtout des pièces diverses. Profitant de certaines relations parisiennes, notamment auprès de Renault, ils s'orientent vers l'automobile et se spécialisent dans la fabrication d'arbres, couronnes, fourchettes et surtout vilebrequins. Ils deviennent des équipementiers de rang 1 et rachètent diverses affaires pour créer SIFCOR, qui regroupe aujourd'hui, outre Courcelles, C2FT à Andrézieux-Bouthéon (Loire) et AMIS (Guéret et Montluçon). SIFCOR assure $20 \%$ de la forge automobile française. Les Forges de Courcelles (430 salariés et 114 millions d'euros de chiffre d'affaires en 2013), toujours sous contrôle familial, investissent dans la robotique et deux énormes presses de 8000 tonnes (2008 et 2014). Placée dans un contexte concurrentiel beaucoup plus rude que celui des prothèses, aggravé encore par la crise actuelle, l'affaire est loin d'atteindre la rentabilité de Marle (résultat net de 900000 euros en 2013).

Le Nogentais Pascal Gillet, à la tête d'une société familiale de près de 10 millions d'euros de chiffre d'affaires (2013), fait aussi partie de ces innovateurs. La société n'a pas le même calibre que les usines précédentes, mais le parcours est tout aussi impressionnant puisque l'on passe de 4 salariés à sa création en 1977 à 70 aujourd'hui ! Cette réussite se nourrit d'une grande capacité d'innovation, qui débouche sur des productions multiples (pinces, coupes câbles, outils pour l'automobile, cisailles...) et de la volonté de trouver des solutions industrielles à des problèmes techniques complexes. L'apport de Gillet réside aussi dans son investissement dans la dynamique collective nogentaise et l'émergence du cluster Nogentech. 
26 Le secteur du médical offre aujourd'hui plus de mille emplois, répartis de manière à peu près équivalente entre Nogent et Chaumont, avec des salaires plutôt élevés. Et ces firmes embauchent. Greatbatch est passé de 240 à 310 salariés en quelques années, Marle de 125 à 168 en dix ans, Gillet de 4 en 1977 à 70 aujourd'hui. C2F Implants, qui n'a que 12 ans, en compte une trentaine. Quant aux spécialistes de la finition des produits HMP et Chesneau, leurs besoins en main-d'œuvre sont considérables. Cette dynamique se traduit par un niveau de chômage contenu : 7,6 \% au second semestre 2014 dans la zone d'emploi Chaumont / Langres, contre 11,2 \% à Saint-Dizier / Vitry-le-François et 10,6 \% en Champagne-Ardenne (INSEE, Fig. 1$)^{9}$. Il faut donc rechercher ce qui a fait la réussite de cette reconversion et plusieurs éléments décisifs peuvent alors être mis en avant.

- Le premier renvoie à ces entrepreneurs qui ont eu très tôt l'intuition d'un marché qui s'ouvrait. Tous sont issus du patronat local (aucun ne vient de l'extérieur) et innovent sur le plan industriel (capacité à répondre à des problèmes techniques, à forger le titane, à créer des outils complexes...) comme sur le plan commercial (cas des Landanger vis-à-vis des chirurgiens). On retrouve là le processus classique de montée en gamme qui permet de contourner la concurrence des pays à bas coûts. Ces entrepreneurs ont aussi joué le rôle de leader en entraînant d'autres sociétés dans leur sillage (Biotech Ortho ou Oury-Guyé pour l'instrumentation médicale...). Ils sont liés par des réseaux d'amitié, trouvent de l'intérêt à se rencontrer et entretiennent, à l'occasion, des liens familiaux (Deguy-Conge et Courcelles par exemple).

- Le second élément concerne le savoir-faire associé au travail des métaux et renvoie à la coutellerie comme à la forge. De la coutellerie, on a utilisé les savoir-faire associés au trempage et aux objets tranchants que l'on retrouve dans les scalpels et les ciseaux de chirurgie, comme dans les lames de sécateur, voire les couteaux pour l'agriculture (rotavators, herses, faucheuses rotatives...). De la forge, on a repris la capacité à innover dans le domaine traditionnel des pièces diverses, celles qui ne concernent pas la coutellerie. Le mouvement a peu à peu engagé certains de ces forgerons vers les prothèses d'articulations, les pièces pour l'automobile (vilebrequins...) ou l'aéronautique (forge de l'aluminium).

29 - La dynamique collective apparait aussi comme une force. Autant les couteliers étaient solitaires, autant ces «nouveaux » entrepreneurs se rencontrent, échangent en passant au-delà des configurations classiques de la concurrence. Cette dynamique d'ensemble a permis l'émergence du cluster Nogentech or, qui dit cluster dit échanges, réunions, partage d'expériences, montage de projets...

- Reste, au moins au départ, un environnement général porteur: les Trente Glorieuses, des marchés en croissance, l'ouverture de l'économie, la réceptivité des élus locaux aussi qui réalisent, dès le milieu des années 1970, la vaste zone industrielle de Nogent. Au final, c'est une intégration réussie dans la mondialisation pour ces industriels. Marle exporte $47 \%$ de sa production, Courcelles travaille beaucoup pour les constructeurs allemands et Greatbatch et Aesculap ignorent quasiment le marché français! L'ouverture des frontières a donc été positive pour ces entreprises et la région, le système productif ayant su proposer des produits à bonne valeur ajoutée recherchés par le marché.

31 - A l'inverse, notons que cette dynamique s'est mise en place dans un environnement géographique peu favorable : un département sans image, sans grande ville, en recul démographique, un potentiel de main-d'œuvre limité, une préfecture peu connue, une 
ville de Nogent médiocrement équipée en commerces et services, un territoire peu attrayant et assez froid, éloigné des grandes métropoles... En fait, l'esprit d'initiative de quelques entrepreneurs, le savoir-faire des forgerons locaux ... et une autoroute à 20 $\mathrm{km}$ ont suffi à contrecarrer la faiblesse des aménités locales.

\section{La métallurgie aujourd'hui dans le Pays de Chaumont}

Livrons nous maintenant à une analyse à la fois quantitative et qualitative de l'industrie métallurgique du territoire en la replaçant, à l'occasion, dans le cadre plus vaste de la Haute-Marne. On trouve alors une grande palette d'entreprises, diversifiées dans leurs productions, mais qui s'inscrivent presque toutes dans la lignée de la tradition forgeronne.

\section{La coutellerie / cisellerie}

Déclinante, voire moribonde, elle ne vaut que par quelques artisans et le Groupe Nogent ${ }^{* * *}$ (voir plus haut).

\section{La tréfilerie}

Cette activité s'appuie sur une longue tradition locale, maintenue par la Tréfilerie de Manois, forte d'une soixantaine de salariés et contrôlée par Arcelor Mittal, le leader mondial de la sidérurgie. La fabrication de ressorts se rapproche de la tréfilerie, surtout quand il s'agit de ressorts à boudins, avec ici deux unités d'une quinzaine de personnes: Ferroflex à Arc-en-Barrois (Groupe allemand Gutekunst) et les Ressorts haut-marnais à Doulaincourt-Saucourt.

Fig. 3 : Les entreprises de la métallurgie dans le Pays de Chaumont

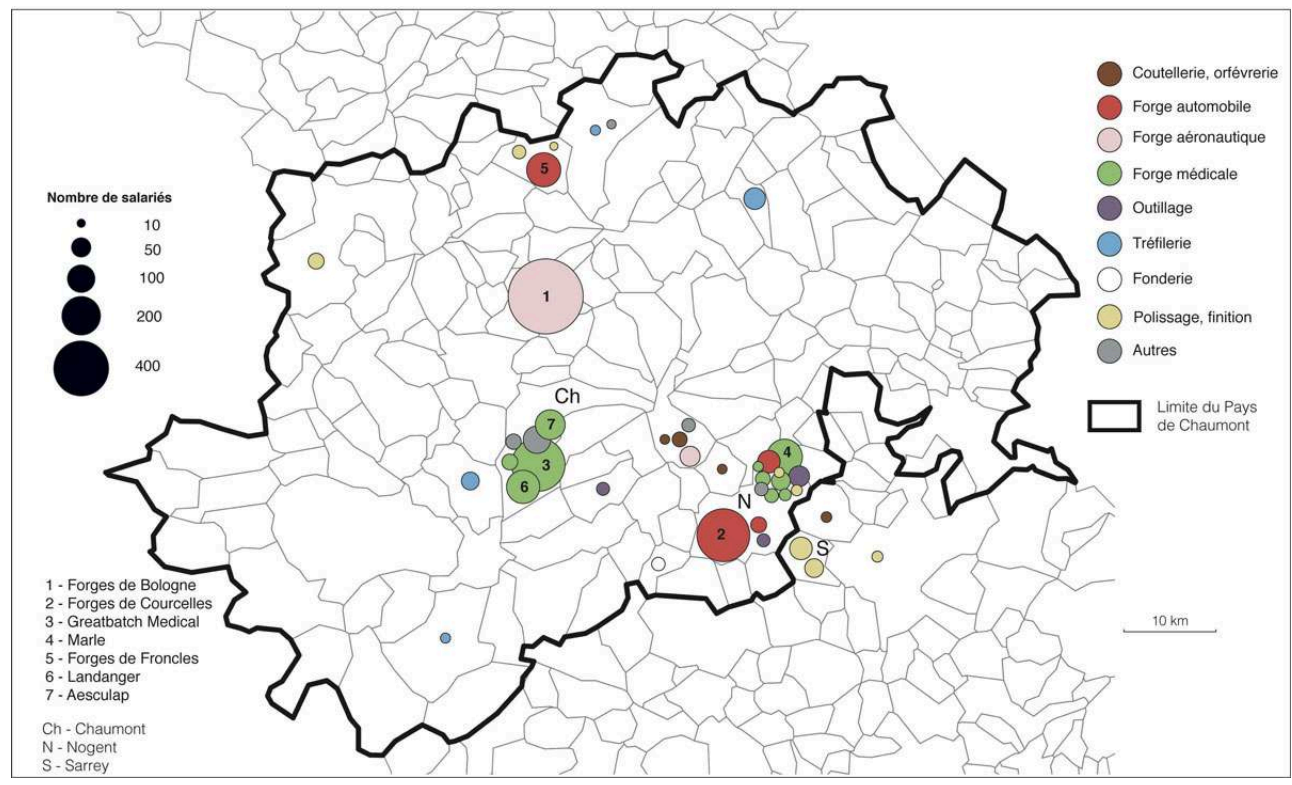

Source : CERAMAC, 2015, d'après enquêtes. 


\section{La permanence de la forge} coutellerie. Elle fait la spécificité de ce territoire alors que la fonderie n'intéresse ici que la CERI à Versaignes-sur-Marne. Elle a subi de profondes restructurations / mutations ces dernières décennies, avec une nette concentration des entreprises et une recherche constante de gains de productivité qui stimule le recul des emplois. Cette forge s'appuie souvent sur des installations de plusieurs centaines de salariés : Bologne, Froncles, Courcelles... Mais les forgerons ont surtout beaucoup évolué dans leurs pratiques et leurs produits. Initialement spécialisés dans la fabrication de pièces pour la coutellerie, ils ont précocement investi dans les pièces diverses. L'intérêt de l'analyse réside alors dans la diversité des options choisies par ces forgerons.

\section{a. Les forgerons de l'automobile}

Orientés vers la fabrication de pièces pour l'automobile, ils perpétuent la tradition des pièces diverses et sont devenus des équipementiers automobiles. lourds, ferroviaire et machinisme agricole. Il est implanté en Pologne (Rzeszow), dans les Ardennes (Monthermé) et à Nogent (Forges de la Ville) et appartient à une structure intermédiaire contrôlée par des capitaux américains. Le Groupe, dirigé depuis Monthermé, emploie une centaine de salariés à Nogent, suite à la reprise des Forges Belligné en 2014.

Tous ces équipementiers sont, globalement, des entreprises de bonne taille et opèrent sur des marchés très concurrentiels. Leur ouverture vers l'international (là où sont les grands constructeurs) est nette, mais leur rentabilité reste faible, surtout depuis la crise de 2008.

\section{b. Les forgerons de l'aéronautique}

41 Leur marché est un peu moins difficile et davantage porteur que celui de l'automobile, mais la logique industrielle est la même que chez les équipementiers et les clients sont aussi des géants mondiaux, constructeurs (Airbus, Boeing, Dassault, Embraer...) ou motoristes (Safran / Snecma...). Une rupture importante avec la tradition réside ici dans le forgeage dominant de pièces en aluminium ou en titane, plus légères que l'acier mais plus techniques.

ForgeAvia, créée en 1871 à Biesles, a progressivement abandonné son environnement coutelier pour se spécialiser dans l'aviation en 1996. Elle compte aujourd'hui 55 
salariés, fait de la sous-traitance pour les constructeurs mondiaux et est particulièrement réputée et dynamique.

Mais l'entreprise la plus emblématique reste les Forges de Bologne, passées au stade industriel dès le début du XXe siècle sous l'impulsion des Vella-Ferrand, des maîtres de forge locaux. Orientées dès 1916 / 1917 vers la forge de l'aluminium pour l'aviation, elles travaillent dans un univers de pièces techniques où le processus de sélection des fabricants se fait par l'intermédiaire de la maîtrise des savoir-faire, de la disponibilité de l'outillage et de la capacité à respecter les délais, à s'inscrire dans la supply chain. Le monde des sous-traitants se consolide actuellement et on évolue pour partie vers des relations de type constructeurs automobiles / équipementiers, sachant qu'il est toutefois plus difficile d'être sous-traitant aéronautique, un métier très exigeant en capitaux et où la procédure de qualification des pièces est très longue. Les Forges de Bologne sont spécialisées dans les aubes de réacteurs.

Suite au retrait des Vella-Ferrand vers 1985, l'affaire change plusieurs fois de mains pour atterrir dans celles de quatre fonds successifs, à partir de 1998 ! En 2013, Sun Capital Partners (50 milliards de dollars de CA) scinde sa filiale Manoir en trois branches et c'est finalement LISI qui reprend Manoir Aerospace (dont les Forges de Bologne) en 2014. Ce groupe discret a ses racines à Delle et Belfort, travaille pour l'automobile, l'aéronautique et accessoirement le médical et est majoritairement contrôlé par les familles fondatrices Kohler, Japy, Veillard et Peugeot. Ces industriels souhaitent renforcer leur branche aéronautique où la consolidation est en cours. Les Forges sont le plus gros site industriel du département, avec 769 salariés, dont 620 en CDI. Elles vendent à $75 \%$ en France où se situent nombre de leurs clients constructeurs ou motoristes.

\section{c. Les forgerons du médical}

Cette industrie doit presque tout aux Landanger et à B. Marle. Les plus grosses usines sont à Chaumont hormis, bien entendu, Marle à Nogent. On trouve aussi des PME de quelques dizaines de personnes, souvent nogentaises (Lasserteux, Biotech Ortho qui fabrique des vis et des plaques pour la réparation des fractures, Oury Guyé qui emploie cinquante personnes et C2F Implants). Des artisans gravitent autour de cette filière qui occupe près de mille emplois, jusqu'à un fabricant de paniers de stérilisation (Plystec Médical) ayant trouvé là une niche spécifique.

\section{d. Les forgerons de l'outillage}

46 Plusieurs fabricants locaux de pièces diverses se sont spécialisés dans l'outillage. Certains sont performantes et en croissance comme le spécialiste des outils à main Gillet et le producteur de lames et couteaux pour l'agriculture Deguy Conge de Luzysur-Marne.

\section{Les spécialistes de la finition des pièces}

47 En aval des forgerons (et des industriels du médical), plusieurs entreprises œuvrent à la finition des produits. La plus dynamique est Chesneau à Sarrey, une société créée en 1965, qui se diversifie un peu par hasard dans l'aéronautique, investit lourdement à partir de la fin du siècle et connaît aujourd'hui une croissance spectaculaire dans le 
polissage (85 salariés). D'autres affaires relèvent de ce créneau comme HMP (Marle) à Sarrey, Alpha Laser à Colombey (marquage, gravure, décoration, 35 salariés) ou encore Raclot et Bodycote à Nogent (traitement de surfaces, 15 salariés chacun). C'est le secteur industriel le plus dynamique du territoire, confronté à de sérieux problèmes de recrutement de main-d'œuvre.

\section{L'industrie du Pays de Chaumont : un cluster industriel ?}

L'industrie est une activité mondialisée qui s'inscrit dans un contexte de compétition très forte entre les entreprises et les territoires. Les spécialistes de l'industrie ont pour habitude de distinguer plusieurs grands types d'espaces aux caractéristiques spécifiques, selon une typologie reprise dans les publications récentes sur les systèmes productifs (Bost, 2014, Carroué, 2014...). On trouve alors les vieilles régions industrielles en reconversion souvent délicate, les grands pôles industriels des années 1950-1960, les systèmes productifs localisés ou cluster et enfin les espaces industriels technopolitains, à la périphérie des grandes villes bien localisées.

49 L'industrie du Pays de Chaumont ne relève à l'évidence ni des grands pôles industriels, ni de l'industrie technopolitaine. Quelques éléments renvoient aux vieilles régions industrielles, au moins si l'on se place dans le cadre plus vaste d'un département où la métallurgie s'était bien développée sur les ressources locales. Celle-ci n'a toutefois pas engendré de vraie région industrielle comme en Lorraine ou dans le Nord, faute de ressources suffisantes, même si quelques entreprises passèrent au stade industriel dès le XIXe siècle. La région se rapproche en fait surtout des systèmes productifs localisés et a d'ailleurs été labellisée comme cluster en 2002. Il semble toutefois que l'on ait davantage ici une sorte de SPL imparfait plutôt qu'un vrai District Industriel à l'italienne.

Ce territoire est bien marqué par une concentration spatiale d'industries manufacturières spécialisées (prothèses, instruments chirurgicaux) et l'émergence, puis l'épanouissement de ce secteur d'activité sont clairement de nature endogène (Merenne-Schoumaker, 2011). On s'appuie ici sur des savoir-faire anciens (issus de la coutellerie et de la forge) ayant généré un artisanat omniprésent, qui a certes périclité, mais a été renouvelé par quelques leaders qui, à force d'innovation, ont su le faire passer au stade industriel. On est là en plein dans un processus de renouveau des productions artisanales (Merenne-Schoumaker, 2011), ou plutôt de réorientation réussie de ces dernières. Le territoire a su se spécialiser dans des produits originaux, à bon contenu technologique et à forte valeur ajoutée, destinés à des marchés en croissance et, qui plus est, peu sensibles à la crise. On est par ailleurs dans un espace « intermédiaire » (Houssel, 1995), mi rural et mi urbain, où l'ambiance est assez propice à l'entreprise (image de l'entrepreneur, valorisation de la créativité, population ouvrière plutôt docile...), et où des réseaux de solidarité préexistants ont été réactivés dans le cluster Nogentech. Bref, cette région s'est développée sur la base de ses ressources propres qui sont ici des ressources humaines: des innovateurs, des entrepreneurs, une main-d'œuvre industrieuse... Ce contexte de développement endogène rejoint le processus qui conduit au système productif localisé. 
51 Mais ce même SPL apparaitt incomplet et sujet à discussion. Son envergure reste limitée, avec quelques milliers d'emplois seulement, sur un territoire assez vaste (une cinquantaine de communes) et une spécialisation productive relative (médical, outillage, équipement automobile...), sauf à considérer que la colonne vertébrale de l'ensemble est la forge, davantage que le médical. Autre point, les entreprises n'opèrent pas entre elles une réelle division du travail. Dès lors, la logique de coopération / concurrence / émulation (Merenne-Schoumaker, 2011) qui est à la base des districts industriels italiens, ne fonctionne qu'à moitié. Concurrence: assez peu car chacun conserve souvent son créneau spécifique (cas des fabricants d'outillage à main), alors que la croissance du marché des prothèses et des instruments de chirurgie est telle que la notion de concurrence est relative. Emulation : certainement, surtout à Nogent, en lien avec des entrepreneurs qui se connaissent et se côtoient, ce phénomène n'étant pas remis en cause par l'arrivée de capitaux étrangers. Coopération: assez peu, compte tenu surtout de la spécialisation de chacun (coupe-cigare chez l'un, vis et plaques médicales chez l'autre, lames agricoles ailleurs...). Autre point, l'industrie locale n'entre dans aucun des cinq grands types de districts industriels définis par Markusen (2000) et repris par d'autres auteurs. Le district nogentais n'est pas à ancrage public, ni structuré par des filiales de grands groupes, ni dominé par quelques grands firmes intégrées verticalement. On se rapprocherait davantage du district marshallien, fondé sur un regroupement de petites entreprises locales, que du district italianisé où la coopération et le rôle de l'administration locale sont plus forts (Merenne-Schoumaker, 2011). Si le district industriel apparaît imparfait, il existe toutefois bel et bien.

La dynamique collective est à analyser au regard du comportement traditionnel de l'artisanat local. Ce dernier s'était toutefois fédéré via la Chambre syndicale de la coutellerie, puis le Syndicat Mixte du bassin d'emploi de Nogent (fin des années 1990) et autour de la marque Nogent sélect (qui sera un échec). Les couteliers avaient aussi constitué un groupement commercial embauchant des représentants exclusifs à la place des multicartes qui ne donnaient pas satisfaction. Le succès fut immédiat mais des problèmes de gestion et de stratégie conduisirent très vite à l'échec.

53 C'est toutefois dans la foulée que s'enclenche une vraie dynamique collective fondée sur les relations d'interconnaissances entre les entrepreneurs. L'association Nogentech, créée le 22 février 2002, réunit alors 64 entreprises de Nogent et des alentours, spécialisées dans le travail des métaux, bien au-delà donc de la seule coutellerie. Très vite reconnu comme Cluster, Nogentech mène des actions spécifiques visant à l'amélioration du système productif local par le développement de synergies entre les entreprises, participe à la création du Pôle Technologique et à l'arrivée de l'Université Technologique de Troyes (2011) et recrute désormais ses adhérents jusqu'à Langres, Troyes et Nancy. Il est labellisé "grappe d'entreprises » en 2010 et, après une période difficile en 2012 / 2014, la nouvelle équipe collabore activement avec la CCI et fait preuve d'une activité soutenue : échange d'information, visites d'entreprises, mise en relation des grosses entreprises locales avec les PME, réflexion sur la formation, collaboration avec le Pôle technologique, la CCI, le CRITT, l'UTT ou encore Pôle Emploi...

54 On est bien là dans une logique de Cluster, d'une organisation territorialisée qui s'appuie et développe des réseaux d'interdépendance, des synergies de proximité. L'objectif est d'échanger, de fédérer, de mutualiser, pour être plus efficace dans la production et la diffusion des produits. La mise en place de ce Cluster doit beaucoup à 
l'implication de quelques leaders (D. Voillequin, B. Gillet, B. Marle, D. Biguet...), à l'élargissement de la base des adhérents à toute la métallurgie, au soutien des collectivités locales et à une évidente volonté d'échange. Nogentech s'inscrit dans une tendance lourde de réflexion sur les dynamiques industrielles à l'échelle d'un territoire de petite dimension qui s'appuie sur des savoir-faire et une spécialisation des entreprises. C'est un des éléments du succès de l'industrie locale, surtout nogentaise.

L'assise territoriale de cet espace industriel mérite aussi quelques remarques. Chaumont est une ville moyenne (moins de 25000 habitants toutefois), préfecture de surcroît et Nogent est bien une ville au sens de l'INSEE (4 000 habitants), mais l'activité locale relève tout autant de l'industrie en milieu rural. Beaucoup d'entreprises, et souvent les plus grosses sont en effet souvent implantées à la campagne: Bologne, Froncle, Sarrey (en-dehors du pays), Mandres-la-Côte, Biesles, Mannois, voire Nogentle-Bas.

\section{De la dynamique industrielle à l'attractivité industrielle?}

La performance globale de cette industrie rejaillit incontestablement sur le milieu local avec une activité plutôt florissante et un chômage contenu. Cela parvient-il à rendre la région attractive? Et surtout pour les hommes? En d'autres termes, parvient-on à passer de ce dynamisme ambiant à une forme d'attractivité industrielle? Autant de questions clefs dans ces milieux déprimés de la « diagonale du vide ».

\section{Une industrie attractive pour les investisseurs...}

57 Le vieux système coutelier était tenu par des artisans du lieu, qui possédaient les ateliers, travaillaient et vivaient au village. De nos jours, les enquêtes montrent une configuration très différente où nombre d'entreprises sont passées entre des mains extérieures à la région.

Neuf investisseurs étrangers emploient ici 1050 personnes. Ils ont manifestement privilégié les affaires les plus performantes et les plus grosses et les secteurs les plus porteurs, à commencer par le médical, qu'ils gèrent presque totalement. D'autres investisseurs français extérieurs à la région contrôlent également diverses sociétés, mais souvent plus petites, à l'exception notable des Forges de Bologne dont le parcours capitalistique récent fut complexe et chaotique ${ }^{10}$.

On constate donc bien une réelle attractivité du territoire pour les investisseurs extérieurs ${ }^{11}$, qui s'explique incontestablement par le positionnement sur des créneaux spécifiques et rentables. Les successions familiales jouent aussi beaucoup, comme chez Landanger et Marle qui, au changement de génération, vendent leur patrimoine. Celui des Landanger est passé aux Américains de Greatbatch et aux Allemands d'Aesculap, même si la famille continue à investir localement. Marle a cédé $80 \%$ de ses parts au fonds américain Carlyle, tout en continuant à investir au pays, notamment chez Chesneau. Cette configuration révèle aussi la relative rareté des capitaux nationaux susceptibles de s'investir dans de telles PME : on rejoint là une faiblesse structurelle du capitalisme national quand, parallèlement, ce sont justement deux grosses PME allemandes, Altenloh et B. Braun, qui achètent respectivement les Forges de Froncles et Aesculap. 
60 du système productif. Une fois le capital parti à l'extérieur, les dividendes s'éloignent
du territoire selon un mécanisme que certains considèrent comme une spoliation. Mais
ces mêmes investissements confortent à l'évidence les entreprises locales et donc
l'emploi.

Fig. 4 : Le contrôle des entreprises du Pays de Chaumont par les capitaux extérieurs

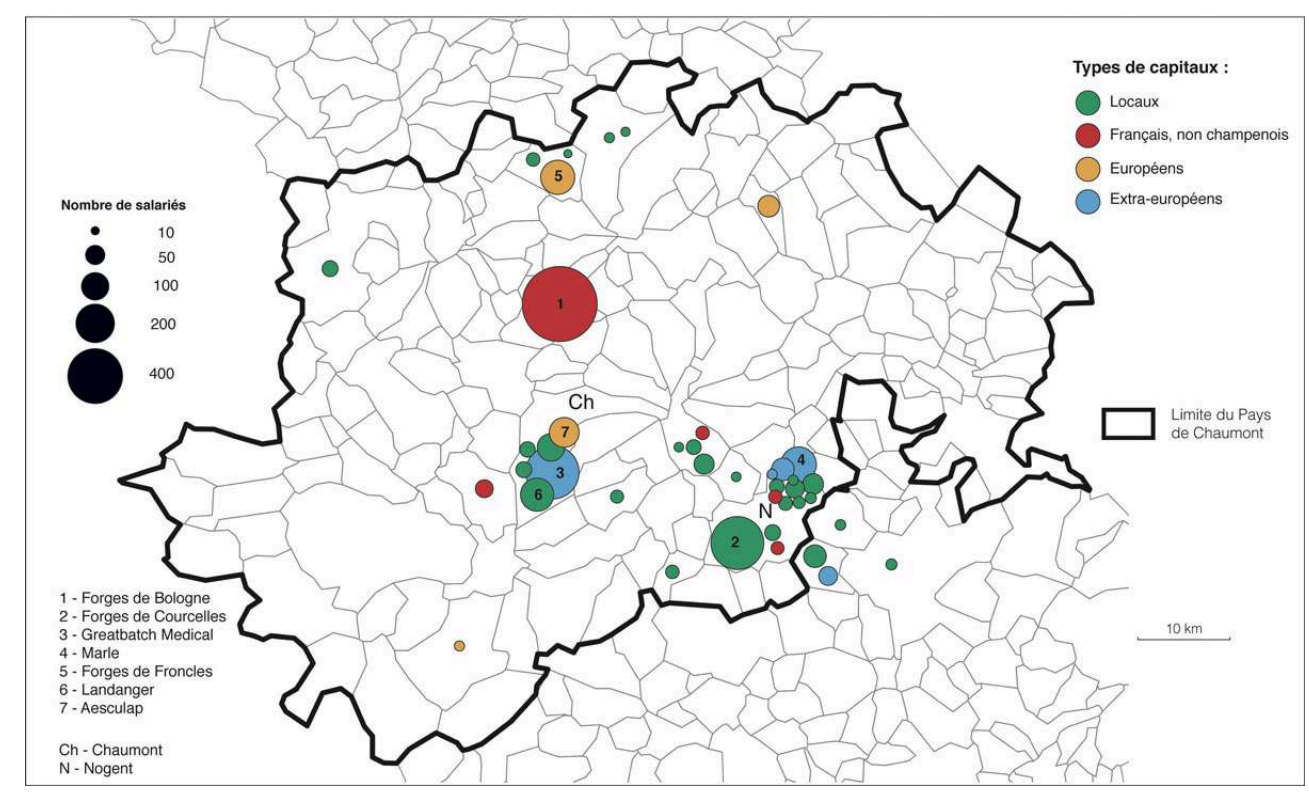

Source : CERAMAC, 2015, d'après Internet et enquêtes.

\section{2. ... beaucoup moins pour les hommes ?}

61 Passons du capital au travail. L'industrie locale est dynamique, crée des richesses et procure de l'emploi, même s'il faut compter avec l'hétérogénéité des situations d'un secteur à l'autre. En 2013, si 12,5 \% des actifs travaillent dans l'industrie (hors BTP) en France, on atteint 16,5 \% en Champagne-Ardenne et 19,2 \% en Haute-Marne, désormais quatorzième département français sur ce plan dans un classement aujourd'hui bouleversé par rapport à la géographie traditionnelle de l'industrie ${ }^{12}$. Plusieurs secteurs embauchent, surtout le médical, davantage lié au vieillissement de la population qu'aux soubresauts de l'économie, avec des créations de poste qui ont plus ou moins compensé le recul de la coutellerie depuis les années 1980. Ces emplois sont, de plus, relativement qualifiés, avec des salaires moyens plus élevés que dans la coutellerie traditionnelle. Le secteur de la finition des pièces est aussi très dynamique, à tel point qu'on nous cite l'exemple d'une entreprise qui dispose d'un horizon de croissance à deux chiffres pour les dix prochaines années!

La forge, automobile ou aéronautique, est en revanche davantage soumise aux impératifs de la productivité et aux soubresauts de l'économie et ses effectifs stagnent ou reculent, les pertes d'emploi étant stimulées par la crise récente de l'automobile. A Bologne, les effectifs sont grossièrement stables (570 « inscrits » (CDI) en 2006 et 620 en 2015), mais on était monté à 900 emplois avant l'an 2000. Froncles a perdu beaucoup d'emplois au cours des dernières décennies. A Courcelles, le travail est fortement soumis à la demande des constructeurs et se maintient difficilement en dépit (ou à 
cause) d'investissements massifs réalisés il y a peu (énormes presses, robotisation massive...). La forge non médicale est donc confrontée à un recul structurel de la maind'œuvre que l'on retrouve dans toute l'industrie française, au-delà des problèmes de compétitivité qui, eux, font reculer les volumes.

Dans le même temps, l'industrie locale n'attire guère les actifs, en dépit d'un taux de chômage assez faible et de possibilités d'emploi, surtout dans le domaine de la finition des produits. Le polissage notamment exige un grand savoir-faire (difficilement reproductible par la machine) et une longue expérience, alors que le système éducatif produit peu de travailleurs qualifiés et que la société se détourne de ce type de métier jugé peu valorisant... Une centaine de postes seraient à pourvoir en dépit de salaires attractifs ${ }^{13}$ et certaines entreprises refuseraient des marchés faute de polisseurs, ce qui a conduit Nogentech, Pôle Emploi, le lycée de Langres et le GRETA de Haute-Marne à mettre en place des formations spécifiques en 2015.

La dynamique industrielle locale n'atteint donc pas la dynamique de certains clusters italiens où émerge une sorte de développement autonome: l'industrie y créé des richesses, stimule les activités périphériques (commerces, artisanat, sous-traitance, encadrement, voire recherche) et, au final, attire de nouvelles populations. Une telle dynamique ne se retrouve pas ici. Le Pays, comme toute la Haute-Marne, continue à perdre des jeunes et attire difficilement les cadres. Les questions d'image sont rapidement sous-jacentes, dans ces plateaux agricoles sans grandes aménités, froids et éloignés des grandes métropoles, même si le carrefour autoroutier de Langres met la région à deux heures de Paris, de Lyon ou de Bâle.

\section{Conclusion}

Le Pays de Chaumont apparaît comme un territoire de faibles densités et toujours en proie au recul de la population : c'est un territoire en marge, en Champagne-Ardenne comme dans le contexte plus vaste du Bassin parisien, une partie de la «diagonale du vide» des géographes. C'est dans le même temps un vrai territoire industriel, qui s'appuie sur une longue tradition de travail des métaux organisée autour de la forge et de la coutellerie. L'intérêt de l'analyse vient certainement du processus de mutation / adaptation qui a eu lieu depuis la guerre, et surtout depuis les années 1980, lorsque le secteur fut confronté de plein fouet à la mondialisation de l'économie. Beaucoup d'entreprises, souvent artisanales, disparaissent, conduisant à un repli massif, à une quasi disparition de la coutellerie / cisellerie qui avait pourtant fait la réputation de la région. Dans le même temps, d'autres se reconvertissent ou émergent en explorant de nouveaux créneaux, qui ont la particularité d'être issus de la forge : l'instrumentation médicale et les implants orthopédiques. Trente ans après, le processus structurel d'adaptation a manifestement réussi. Il s'explique fondamentalement par le rôle des hommes : dynamisme de quelques entrepreneurs, savoir-faire des forgerons, héritage de l'histoire, importance du collectif... Cette dynamique a été créatrice d'emploi et contribue aujourd'hui à la faiblesse relative du chômage dans le territoire, mais reste mal connue en dehors de la Haute-Marne, ce qui renvoie à des faiblesses structurelles locales en matière de communication, de capacité à communiquer sur ses propres réussites. 
Les entreprises du secteur de métallurgie du Pays de Chaumont en 2015 (Entreprises de plus de 10 salariés)

\begin{tabular}{|c|c|c|c|c|c|}
\hline Entreprise & \begin{tabular}{|l} 
Localisation \\
\end{tabular} & \begin{tabular}{|c}
$\begin{array}{c}\text { Nombre } \\
\text { de } \\
\text { salaries }\end{array}$ \\
\end{tabular} & Activités & \begin{tabular}{|c|c|c|c|c|c|} 
Capital \\
(si exterieur)
\end{tabular} & Divers \\
\hline 1-Aesculap & Chaumont & 120 & $\begin{array}{l}\begin{array}{l}\text { Instruments de } \\
\text { chirungie }\end{array} \\
\end{array}$ & \begin{tabular}{|l|}
$\begin{array}{l}\text { Groupe } \\
\text { allemand B. } \\
\text { Braun }\end{array}$ \\
\end{tabular} & \\
\hline 2-Alpha laser & $\begin{array}{l}\text { Colombey- } \\
\text { les-Deux- } \\
\text { E-lises }\end{array}$ & 35 & $\begin{array}{l}\text { Marquage, gravure, } \\
\text { decoration... }\end{array}$ & & \\
\hline 3-APPA & Nogent & 25 & Usinage, soudure & \begin{tabular}{|l|} 
Groupe \\
Chanitec \\
(Champlitte)
\end{tabular} & \\
\hline $\begin{array}{l}\text { 4-Applications } \\
\text { Industrielles du } \\
\text { Vide }\end{array}$ & Froncles & 10 & \begin{tabular}{|l|} 
Traitement et \\
revêtement des métaux
\end{tabular} & & $\begin{array}{l}\text { Mème } \\
\text { actionariat } \\
\text { que Clas } \\
\text { Galvaplast }\end{array}$ \\
\hline 5-Attifl & \begin{tabular}{|l|l} 
Semoutiers \\
\end{tabular} & 43 & $\begin{array}{l}\text { Fil métalliquque (auto, } \\
\text { agriculture. industrie, } \\
\text { fermetures de } \\
\text { bocaux...) }\end{array}$ & \begin{tabular}{|l|} 
Groutpe \\
Arnetal \\
(Semoutiers, \\
Hallunin, \\
Tuluisie)
\end{tabular} & \\
\hline 6-Atelier Bois & \begin{tabular}{|l|} 
Chaumont \\
\end{tabular} & 102 & $\begin{array}{l}\begin{array}{l}\text { Ossatures métalliques. } \\
\text { couverture. } \\
\text { sernurerie... }\end{array} \\
\end{array}$ & & \\
\hline 7-Biotech Ortho & \begin{tabular}{|l|l|} 
Nogent \\
\end{tabular} & 19 & \begin{tabular}{|l}
$\begin{array}{l}\text { Vis et plaques } \\
\text { orthopediques }\end{array}$ \\
\end{tabular} & \begin{tabular}{|l|l|}
$\begin{array}{l}\text { Groupe US } \\
\text { Wright } \\
\text { Medical }\end{array}$ \\
\end{tabular} & \\
\hline 8-Bodycote & Nogent & 16 & \begin{tabular}{|l|} 
Traitement des aciers \\
\end{tabular} & & \\
\hline 9-C2F Implants & Nogent & 30 & 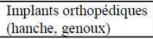 & Landanger & \\
\hline 10-CERI & \begin{tabular}{|l|l} 
Versaignes- \\
\end{tabular} & 26 & Fonderie & & \\
\hline $\begin{array}{l}11-\text { Chesneau Ch. } \\
\text { et fils }\end{array}$ & \begin{tabular}{|l} 
Sarrey \\
\end{tabular} & 70 & $\begin{array}{l}\text { Polissage et auttes } \\
\text { travaux de finition des } \\
\text { pièces métalliques. }\end{array}$ & & Hors Pays \\
\hline $\begin{array}{l}\text { 12-Clas } \\
\text { Galvaplast }\end{array}$ & Froncles & 25 & $\begin{array}{l}\text { Traitements de surface } \\
\text { (nickelage. } \\
\text { chromage...), revente } \\
\text { de méraux } \\
\end{array}$ & & $\begin{array}{l}\text { Même } \\
\text { actionnariat } \\
\text { qu'AVV }\end{array}$ \\
\hline $\begin{array}{l}\text { 13-Daguin } \\
\text { estampage }\end{array}$ & \begin{tabular}{|l} 
Is-en- \\
Bassigny \\
\end{tabular} & 14 & \begin{tabular}{|l} 
Coutellerie Cisellerie \\
\end{tabular} & & \\
\hline 14-Deguy Conge & \begin{tabular}{|l|} 
Lusizy Marme \\
\end{tabular} & 23 & $\begin{array}{l}\text { Lames agricoles } \\
\end{array}$ & & \\
\hline $\begin{array}{l}\text { 15-Landanger } \\
\text { Delacroix } \\
\text { Chevalier }\end{array}$ & \begin{tabular}{|l|} 
Chaumont \\
\end{tabular} & 150 & $\begin{array}{l}\begin{array}{l}\text { Instruments de } \\
\text { chirurgie }\end{array} \\
\end{array}$ & \begin{tabular}{|l} 
Landanger \\
\end{tabular} & \\
\hline 16-Eloi Pernet & Nogent & 27 & $\begin{array}{l}\text { Coutellerie de luxe, } \\
\text { commerce de } \\
\text { coutellerie }\end{array}$ & $\mathrm{CDC}$ & \\
\hline 17-Ferronex & \begin{tabular}{|l}
$\begin{array}{l}\text { Arc-en- } \\
\text { Banois }\end{array}$ \\
\end{tabular} & 14 & \begin{tabular}{|l|l} 
Fabrication de ressorts \\
\end{tabular} & \begin{tabular}{|l|}
$\begin{array}{l}\text { Groulpe } \\
\text { Gutekumst } \\
\text { (All) }\end{array}$ \\
\end{tabular} & \\
\hline 18-Forgeavia & \begin{tabular}{|l|l|} 
Biesles \\
\end{tabular} & 55 & $\begin{array}{l}\text { Forge à chaud pour } \\
\text { l'aviation, fabrication } \\
\text { d'outils pour la forge }\end{array}$ & & \\
\hline $\begin{array}{l}\begin{array}{l}\text { 19-Forge } \\
\text { Béligné }\end{array} \\
\text { (a) }\end{array}$ & Nogent & 35 & $\begin{array}{l}\text { Forge, estampage de } \\
\text { precision. }\end{array}$ & $\begin{array}{l}\text { Groupe } \\
\text { Forgex- }\end{array}$ & \\
\hline
\end{tabular}

\begin{tabular}{|c|c|c|c|c|c|}
\hline & & & parachèvement. & $\begin{array}{l}\text { Raguet depuis } \\
2014\end{array}$ & \\
\hline 20-Forges de & \begin{tabular}{|l|} 
Bologne \\
\end{tabular} & 769 & \begin{tabular}{|l|l} 
Forge pour \\
\end{tabular} & \begin{tabular}{|l|l|l} 
Groupe LSI \\
\end{tabular} & \\
\hline $\begin{array}{l}\text { Bologite } \\
\text { 21-Forges } \\
\text { Correelles }\end{array}$ & Nogent & 430 & 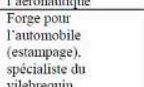 & $\begin{array}{l}\text { Gepusipe } \\
\text { franceais } \\
\text { SIFCOR }\end{array}$ & Famille Deguty \\
\hline $\begin{array}{l}\text { 22-Forges de } \\
\text { Froncles }\end{array}$ & \begin{tabular}{|l|l|} 
Froncles \\
\end{tabular} & 160 & $\begin{array}{l}\text { Forge à froid pour } \\
\text { lautomobile (écrous, } \\
\text { vis...) }\end{array}$ & \begin{tabular}{|l} 
Groupe \\
Altenloh (All)
\end{tabular} & \\
\hline $\begin{array}{l}\text { 23- Forgex } \\
\text { Raguet }\end{array}$ & Nogent & 70 & Forge à chaud & \begin{tabular}{|l|} 
Groupe \\
Forgexp puis \\
Metcap, sous \\
contróle US
\end{tabular} & $\begin{array}{l}\begin{array}{l}\text { Forges de la } \\
\text { Ville }\end{array} \\
\end{array}$ \\
\hline 24-Gillet Group & Nogent & 60 & $\begin{array}{l}\text { Conception et } \\
\text { fabrication doutlilage } \\
\text { à main. dourils } \\
\text { spéciax et de } \\
\text { machines speciales. }\end{array}$ & & \\
\hline $\begin{array}{l}\text { 25-Gorse } \\
\text { Mécaumique } \\
\text { Génerale }\end{array}$ & Biesles & 25 & $\begin{array}{l}\text { Outillage de forge. } \\
\text { mécanique génerale }\end{array}$ & $\begin{array}{l}\text { Groupe } \\
\text { Chanitec } \\
\text { (Champline) }\end{array}$ & \\
\hline $\begin{array}{l}\text { 26-Greatbatch } \\
\text { medical }\end{array}$ & Charmont & 350 & $\begin{array}{l}\begin{array}{l}\text { Prothèeses } \\
\text { orthopediques }\end{array} \\
\end{array}$ & 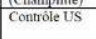 & \\
\hline $\begin{array}{l}\text { 27-Hante-Marne } \\
\text { Parache-yement }\end{array}$ & Sarrey & 48 & $\begin{array}{l}\text { Traitementit de surfface. } \\
\text { palissog }\end{array}$ & \begin{tabular}{|l} 
Groupe Marle \\
\end{tabular} & Hors Pays \\
\hline 28-KImex & \begin{tabular}{|l} 
Biesles \\
\end{tabular} & 12 & $\begin{array}{l}\text { Positeleterie non } \\
\text { fermante }\end{array}$ & $\begin{array}{l}\text { Méme groupe } \\
\text { que } \\
\text { Nogento *t }\end{array}$ & \\
\hline 29-Lasserteux & Nogent & 14 & $\begin{array}{l}\text { Instruments de } \\
\text { chinurgie. }\end{array}$ & & \\
\hline 30-Maire Didier & \begin{tabular}{|l|} 
Sarrey puis \\
Chaumont
\end{tabular} & 35 & $\begin{array}{l}\text { Instruments de } \\
\text { clinurie. }\end{array}$ & Landanger & $\begin{array}{l}\text { Déménagemenen } \\
\text { a Chaument en } \\
2014\end{array}$ \\
\hline 31--Marle & Nogent & 170 & $\begin{array}{l}\text { Prothèses } \\
\text { orthopédiques }\end{array}$ & $\begin{array}{l}\text { Fonds Carlyle } \\
\text { (USA) a } 80 \%\end{array}$ & \\
\hline $\begin{array}{l}\text { 32-Manu. pour } \\
\text { l'Extrusion } \\
\text { d'Alliage }\end{array}$ & \begin{tabular}{|l|}
$\begin{array}{l}\text { Doulaincourt- } \\
\text { Saucourt }\end{array}$ \\
\end{tabular} & 12 & $\begin{array}{l}\text { Forrege, produitis en } \\
\text { laiton et cuive }\end{array}$ & & \\
\hline 33-Mécadis & \begin{tabular}{|l} 
Mandires \\
Bijsestes
\end{tabular} & $\frac{12}{31}$ & \begin{tabular}{|l} 
Orfestrerie, argenture \\
Coutenllerie.
\end{tabular} & & \\
\hline 35-Oury Guyé & \begin{tabular}{|l|} 
Nogent \\
\end{tabular} & $\frac{11}{48}$ & $\begin{array}{l}\text { lustruments de } \\
\text { chinurie }\end{array}$ & & \\
\hline 36-Outils PAM & Nogent & 23 & Outillage à main & \begin{tabular}{|l|} 
Groupe \\
Devillé \\
(Mane et \\
Loire)
\end{tabular} & \\
\hline 37. Poll 2000 & $\begin{array}{l}\text { Val de Meuse } \\
\text { Vil }\end{array}$ & 15 & Polissage & & \\
\hline $\begin{array}{l}\text { 38-Raclot } \\
\text { 39-Ressorts }\end{array}$ & $\begin{array}{l}\text { Nogent } \\
\text { Doulaincourt- }\end{array}$ & $\frac{14}{14}$ & $\begin{array}{l}\text { Traitement de surfaces } \\
\text { Ressorts, pieces en fil }\end{array}$ & & \\
\hline $\begin{array}{l}\text { hyaut-marnais } \\
\text { ha-Soremo }\end{array}$ & $\begin{array}{l}\text { Salcoumt } \\
\text { Chaumont }\end{array}$ & 33 & $\begin{array}{l}\text { cambre } \\
\text { Recyclage de moteurs. } \\
\text { fabrication dalliages }\end{array}$ & & \\
\hline $\begin{array}{l}\text { 41-Trénllerie de } \\
\text { Manois }\end{array}$ & Mannois & 63 & Cables et 1 & \begin{tabular}{|l|} 
Groupe \\
Arcelor Mittal
\end{tabular} & $\begin{array}{l}\begin{array}{l}\text { Filiale Wire } \\
\text { Solutions }\end{array} \\
\end{array}$ \\
\hline
\end{tabular}




\section{BIBLIOGRAPHY}

Bazin M., 2014, Patrimoine industriel et identité territoriale dans les Ardennes, Territoire en mouvement, Revue de géographie et aménagement [En ligne], 21/2014.

Benko G, Lipietz A (dir), 1992, Les régions qui gagnent, districts et réseaux : les nouveaux paradigmes de la géographie économique, Paris, PUF, Coll. Economie en mouvement.

Benko G, Lipietz A (dir), 2000, La richesse des régions. La nouvelle géographie socio-économique, Paris, PUF, Coll Economie en mouvement.

Berton J., 2002, 101 figures haut-marnaises, 190 p.

Borloz P., Gielen P. Dir., 2012, Atlas des développements durables en Champagne-Ardenne, CRDP, Académie de Reims, Préfecture de Région, 192 p.

Bost F., 2014, La France : mutation des systèmes productifs, SEDES, Col. CNED SEDES, 253 p.

Bruno G. (pseudo), 1877, Le tour de la France par deux écoliers, Ed. Belin.

Bulard M., 1904, L'industrie du fer dans la Haute-Marne, Annales de Géographie, vol.13, n 69, p. 223-242 et $n^{\circ} 70$, p. 310-321.

Cardot M., 2013, La forge de Froncles dans l'Histoire XXe siècle, 340 p.

CERAMAC, 2015, Réalisation de l'évaluation ex-post de la politique de développement local 2003 / $2013 \mathrm{du}$ Pays de Chaumont et étude de prospective territoriale pour la définition des axes de la future charte de développement du territoire, $304 \mathrm{p}$.

Carroué L., 2014, La France : les mutations du système productif, A. Colin, Col. U, 240 p.

Collectif, 1997, La métallurgie en Haute-Marne du Moyen Age au XXe siècle, Association pour la valorisation des atouts culturels de Champagne-Ardenne, $310 \mathrm{p}$.

Collectif, 2004, Champagne-Ardenne, Bonneton Ed., 320 p.

DATAR, 2001, Réseaux d'entreprises et territoires. Regards sur les systèmes productifs locaux, Paris, La Doc. Française.

Gallois L., 2012, Pacte pour la compétitivité de l'industrie française, La Documentation Française, 74 p.

Garnotel J., 2006, Champagnes au pluriel. Un espace, une histoire, un vin, une région, 314 p.

Gilardot E., 2013, Fonderies en Haute-Marne, une aventure humaine, 152 p.

Gilardot E., 2014, Cœur de forge, 174 p.

Houssel J.-P., 1995, Districts industriels et milieux intermédiaires, Revue de Géographie de Lyon, vol. 70, nº p. 7-10.

Marshall A., 1890, Principes d'économie politique, 544 p.

Markusen A., 2000, Des lieux aimants dans un espace mouvant. Une typologie des districts industriels, in Benko G. et Lipitez A. (dir), La richesse des régions, La nouvelle géographie socioéconomique, Paris, PUF, Coll. Economie en liberté.

Merenne-Schoumaker B., 2011, La localisation des industries. Enjeux et dynamiques, PU Rennes, 264 p. Nonn H., Héraud J.-A. (dir.), 1995, Les économies industrielles en France de l'est, Presses Universitaires de Strasbourg, 305 p. 
Pecqueur B. (dir), 1996, Dynamiques territoriales et mutations économiques, Paris, L'Harmattan.

Région Champagne-Ardenne, 2013, Champagne-Ardenne industries, sous-traitance \& co-traitance 2013/2014, 296 p.

RITMA, sous la direction de Héraud J.-A. et Nonn H., 2001, Regards croisés sur les espaces en marge(s), Presses Universitaires de Strasbourg, 239 p.

Savouret Ph., 1983, La coutellerie nogentaise au XIX ${ }^{e}$ siècle, D. Guéniot ed. Langres, 222 p.

\section{NOTES}

1. - La population rurale du territoire atteint $59,6 \%$ en 2011 selon l'INSEE.

2. - L'industrie du Pays de Chaumont est surtout concentrée dans la préfecture et à Nogent, mais on trouve aussi de nombreuses installations ponctuelles au cœur des campagnes (carte $n^{\circ} 1$ ). Cette activité s'inscrit dans un contexte départemental marqué par une forte emprise de l'industrie : fonderie au Nord (Saint-Dizier, Wassy...) et plasturgie au Sud, à Langres, dans le sillage de Plastic Omnium et de Freudenberg.

3. - La coutellerie / cisellerie serait passée de 1750 emplois en 1975 (Savouret, 1983) à moins de cent aujourd'hui (enquêtes). Cet énorme recul (- $95 \%$ !) a touché une activité de main-d'œuvre et des salariés souvent peu qualifiés, pas toujours faciles à réemployer.

4. - Les données disponibles les plus pertinentes concernent la zone d'emploi qui regroupe les deux arrondissements de Chaumont et de Langres, nettement plus vaste donc que le seul Pays de Chaumont. Notons aussi que les statistiques du chômage visent l'ensemble de l'économie et pas seulement l'industrie.

5. - Eloi Pernet est une vieille coutellerie de Biesles, désormais installée à Nogent, qui se réorienta dès les années 1950 vers les instruments de chirurgie. Avec aujourd'hui 27 personnes, elle n'a conservé qu'une petite fabrication d'objets tranchants haut de gamme (coupes cigares...), fait de la retouche et surtout du commerce de couteaux, ciseaux, instruments de manucure et pinces à ongle. Bref, ce n'est plus un pur coutelier (site internet du Groupe www.eloi.fr).

6. - Une affaire d'une vingtaine de salariés est aujourd'hui fermée.

7. - La fabrication des prothèses exige des techniques complexes. L'articulation de la hanche est bien maîtrisée depuis déjà longtemps. Celle du genou l'est devenue récemment, mais celle de l'épaule reste encore d'une maîtrise délicate.

8. - Wichard, aujourd'hui implanté à Thiers, est le leader mondial de l'accastillage marin. Les Conge contrôlent l'entreprise Deguy-Conge spécialisée dans la production de lames et couteaux agricoles.

9. - Précisons que le processus de départ des jeunes, qui est une réalité dans la région, limite certainement le niveau du chômage...

10. - En 1995, l'usine passe aux mains du métallurgiste français Manoir Industrie qui la revend au géant américain de l'aluminium Alcoa ... qui la cède à des financiers dès 1998. Quatre fonds de pension se succèdent alors (HSBC, Lazard, Fin Active et Sun Capital, via sa filiale Manoir). L'arrivée de LISI signe le retour à un capitalisme familial plus stable.

11. - Cette mondialisation s'exprime aussi par le changement rapide des propriétaires, à l'image de l'usine mère de Landanger, contrôlée successivement par DePuy, le $\mathrm{n}^{\circ} 2$ de la chirurgie orthopédique à l'époque (1997), Johnson \& Johnson (1998), le Suisse Precimed (2007) et Greatbatch (2008), soit quatre changements de propriétaires en 11 ans !

12. - En 2013, les départements les plus industriels sont l'Ain (22,4\% des actifs dans l'industrie, hors BTP), le Jura (21,7\%), la Vendée (21,6 \%), la Haute-Saône et la Mayenne (21\%), le Doubs 
(20,7 \%), la Drôme, l'Eure et les Ardennes (20,5\%), les Vosges $(20,4 \%)$, la Haute-Loire $(20,3 \%)$, l'Orne (19,5\%), la Sarthe (19,3\%) et la Haute-Marne (19,2\%).

13. - Un polisseur qualifié peut envisager 1700 euro net mensuels après deux années de formation / expérience.

\section{ABSTRACTS}

The industrial tradition of the Chaumont' region is that of the forge, like in Nogent, which had early specialized itself in cutlery and more particularly in scissors manufacturing. This powerful craft industry nevertheless failed to adapt and has been engaged in a long movement of decline from the late nineteenth century, as to have less than a hundred jobs today. After the war, blacksmiths however fueled a strong structural shift movement to surgical instruments and surgical implants (artificial prosthesis). The article describes and analyzes this long-term change, by emphasizing the role of enterprises and territorial dynamics at work in this low density area, but marked by the industry. It also reflects the functional characteristics of this local industry by questioning in particular the concept of clusters, in conjunction with the presence here of the Nogentech cluster.

La tradition industrielle de la région de Chaumont est celle de la forge, à l'image notamment de la ville de Nogent, qui s'était précocement spécialisée dans la coutellerie et surtout la cisellerie. Cet artisanat n'a toutefois pas réussi à s'adapter et s'est engagé dans un long mouvement de repli dès la fin du XIXe siècle, au point de compter moins de cent emplois aujourd'hui. Les forgerons ont toutefois alimenté, dès l'après-guerre, un puissant mouvement de réorientation structurelle vers les instruments de chirurgie, puis les implants chirurgicaux (prothèses articulaires). L'article décrit et analyse cette mutation de long terme en insistant sur le rôle des entreprises et les dynamiques territoriales à l'œuvre dans cette région de faible densité, mais marquée par l'industrie. Il réfléchit aussi aux caractéristiques fonctionnelles de cette industrie locale en s'interrogeant notamment sur la notion de cluster, en liaison avec la présence ici du cluster Nogentech.

\section{INDEX}

Mots-clés: Chaumont, cluster, coutellerie, forge, Haute-Marne, Nogent

Keywords: Chaumont, cluster, cutlery, forge, Haute-Marne, Nogent

\section{AUTHOR}

DANIEL RICARD

CERAMAC / Université Blaise Pascal - Clermont-Ferrand - daniel.ricard@univ-bpclermont.fr 